\title{
Effect of Oral Colestimide on the Elimination of High-dose Methotrexate in Patients With Primary Central Nervous System Lymphoma
}

\author{
-Two Case Reports-
}

\author{
Keishi MAKINO, Masato KOCHI, Hideo NAKAmURA, Jun-ichiro KURODA, \\ Yoshiteru HONDA*, Yukitaka USHIO, and Jun-ichi KURATSU \\ Department of Neurosurgery, Kumamoto University School of Medicine, Kumamoto; \\ ${ }^{*}$ Department of Pharmacy, Kumamoto University Hospital, Kumamoto
}

\begin{abstract}
Delayed methotrexate (MTX) elimination occurred in two patients with primary central nervous system lymphoma undergoing high-dose MTX treatment. Oral administration of the anion exchange resin colestimide, which binds MTX effectively in vitro, effectively accelerated MTX elimination. Colestimide probably interrupts the enterohepatic circulation, and is a potential oral antidote to MTX toxicity.
\end{abstract}

Key words: malignant lymphoma, colestimide, methotrexate

\section{Introduction}

Methotrexate (MTX), an inhibitor of dihydrofolate reductase, is widely used in the treatment of various malignant tumors. High-dose MTX administration is particularly effective against primary central nervous system (CNS) lymphoma. ${ }^{1)}$ MTX administered at a dose of $3.5 \mathrm{~g} / \mathrm{m}^{2}$ penetrates the blood-brain barrier and yields sustained therapeutic cerebrospinal fluid levels lasting longer than 24 hours. However, prolonged exposure to low-level $\left(10^{-8} \mathrm{M}\right)$ concentrations of MTX may cause neurotoxic reactions, mainly myelosuppression, gastrointestinal mucositis, and hepatitis. ${ }^{3)}$ Other types of toxicity depend on the administration regimen: chronic low-dose administration has been associated with cirrhosis, interstitial pneumonitis, and osteoporosis whereas high-dose systemic therapy can result in renal dysfunction, vomiting, and acute desquamative dermatitis. Elimination of MTX from the plasma after intravenous injection passes through three phases: distribution, renal clearance, and enterohepatic circulation. ${ }^{11,12,15)}$ The third phase may be implicated in the reported bone marrow and gastrointestinal toxicity. ${ }^{6,7)}$

Received February 15, 2005; Accepted June 13, 2005
Anion-exchange resins effectively reduce cholesterol levels in patients with hypercholesterolemia. Oral administration of cholestyramine, an insoluble quaternary ammonium anion exchange resin, increases the fecal excretion of bile acids, so blocking enteral reabsorption. ${ }^{5)}$ MTX is an anionic drug with a structure containing 2 carboxyl groups and a molecular weight similar to that of bile acids. Therefore, cholestyramine could accelerate MTX elimination in patients experiencing toxicity associated with high-dose MTX therapy by binding MTX in the gut and interrupting the enterohepatic circulation. ${ }^{8)}$ Colestimide, a new type of anion-exchange resin, is reported to effectively absorb MTX.10)

We treated two patients with high-dose MTX administration for primary CNS lymphoma which resulted in the delayed elimination of MTX. Oral administration of colestimide effectively accelerated excretion of the MTX.

\section{Case Reports}

Case 1: A 32-year-old male was treated under a diagnosis of primary CNS lymphoma with three cycles of high-dose MTX $\left(3.0 \mathrm{~g} / \mathrm{m}^{2}\right)$ followed by whole-brain irradiation ( $30 \mathrm{~Gy}$ ) in June 2000 . At that time, no delayed MTX elimination was observed and complete remission was obtained. However, recur- 

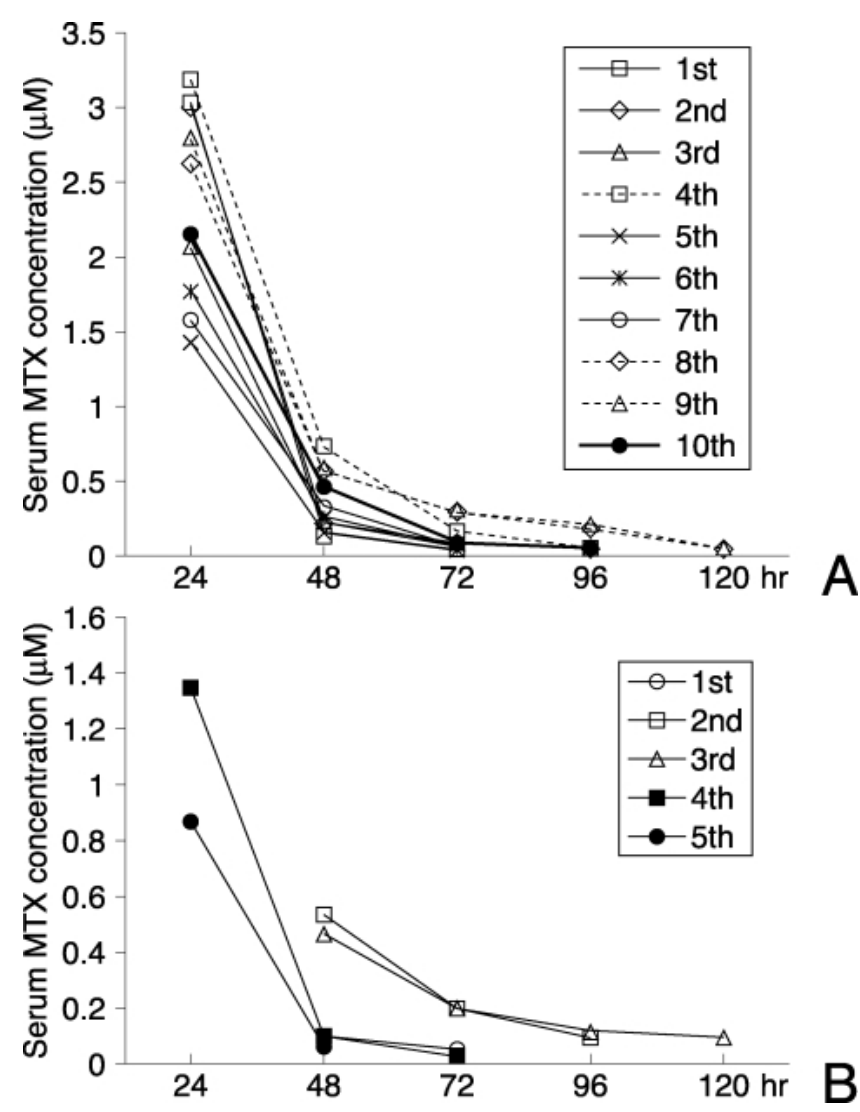

Fig. 1 Serum methotrexate (MTX) concentrations throughout the treatment course in Cases 1 (A) and 2 (B). Colestimide was orally administered during the 10th cycle in Case 1 and the 4th and 5th cycles in Case 2.

rence was noted in September 2001, and he received further cycles of high-dose MTX $\left(3.5 \mathrm{~g} / \mathrm{m}^{2}\right)$ consisting of 3-hour infusion administered on the 1st day of the treatment. Urine alkalinization was started with sodium bicarbonate before the start of MTX infusion and citrovorum factor rescue was begun at 24 hours after the beginning of MTX infusion. Serum MTX levels were measured at 24,48 , and 72 hours after the start of MTX infusion. Although the recurrent tumor disappeared after the first treatment, maintenance treatment was continued for 1 year. After the 4th, 8th, and 9th cycles, delayed MTX elimination was noted (Fig. 1A), but he manifested no clinical signs of toxicity other than nausea and appetite loss. There was no bone marrow toxicity and serum creatinine level was within the normal range. Leucovorin rescue was continued until the serum MTX level dropped below $0.1 \mu \mathrm{mol} / \mathrm{l}$. Since delayed MTX elimination occurred after the 8th and 9th cycles, oral colestimide (3 g/day) was administered on days 1-3 of the 10th cycle. This resulted in accelerated MTX excretion and serum MTX level was below $0.1 \mu \mathrm{mol} / \mathrm{l}$ at 72 hours after the start of MTX administration (Fig. 1A).

Case 2: A 49-year-old male was treated under a diagnosis of primary CNS lymphoma with a total of five cycles of high-dose MTX $\left(3.5 \mathrm{~g} / \mathrm{m}^{2}\right)$ in December 2001. After the 2nd and 3rd cycles, delayed MTX elimination was noted (Fig. 1B). He experienced nausea and appetite loss and manifested elevation of glutamic oxaloacetic and glutamic pyruvic transaminase, but no bone marrow suppression was noted and serum creatinine level was within the normal range. To accelerate MTX excretion, oral colestimide ( $3 \mathrm{~g} /$ day) was administered on days 1-3 of the 4th and 5th cycles. His serum MTX level was below $0.1 \mu \mathrm{mol} / \mathrm{l}$ at 72 and 48 hours after the start of MTX administration, respectively (Fig. 1B).

\section{Discussion}

Renal MTX clearance decreases as the serum concentration of MTX increases, ${ }^{13)}$ so the drug elimination delay becomes longer with higher administered dose. Since prolonged exposure to low levels of MTX induces neurotoxic reactions, ${ }^{3}$ the elimination of MTX must be accelerated. MTX levels were higher in the bile than the plasma of patients. ${ }^{4)}$ MTX concentration in jejunal aspirate obtained 4 to 5 days after MTX administration was higher than in serum obtained at 24 hours after administration. ${ }^{2)}$ These observations suggest that biliary and possibly enteral secretion and reabsorption contribute to the slow third phase of MTX elimination.

Oral administration of activated charcoal reportedly reduced the serum MTX levels in the second elimination phase. ${ }^{9)}$ This may be due to adsorption of biliary excreted MTX which is consequently not reabsorbed enterally. Oral cholestyramine facilitates MTX excretion in the enterohepatic circulation ${ }^{8,14}$ without affecting the serum MTX level at 24 hours after infusion, which may reflect the renal elimination of the drug ${ }^{8)}$ and suggests that cholestyramine may improve the total body clearance of MTX by enhancing extrarenal excretion.

We administered colestimide to our patients because this agent has greater adsorptive capacity than cholestyramine for bile acids and MTX in vitro. ${ }^{10)}$ Oral colestimide accelerated MTX elimination without producing any side effects, so provides a simple, safe method for the control of serum MTX levels in patients with malignant CNS tumors. 


\section{References}

1) Abrey LE, Yahalom J, DeAngelis LM: Treatment for primary CNS lymphoma: the next step. J Clin Oncol 18: 3144-3150, 2000

2) Baird GM, Dossetor JF: Methotrexate enteropathy. Lancet 1: 164, 1981

3) Bleyer WA, Drake JC, Chabner BA: Neurotoxicity and elevated cerebrospinal-fluid methotrexate concentration in meningeal leukemia. $N$ Engl J Med 289: 770-773, 1973

4) Calvert AH, Bondy PK, Harrap KR: Some observations on the human pharmacology of methotrexate. Cancer Treat Rep 61: 1647-1656, 1977

5) Carey JB Jr: The altered distribution of the bile acid pool in obstructive jaundice and its correction including relief of pruritus with a bile acid sequestering resin. J Clin Invest 40: 1028, 1961

6) Chabner BA, Johns DG, Bertino JR: Enzymatic cleavage of methotrexate provides a method for prevention of drug toxicity. Nature 239: 395-397, 1972

7) Chabner BA, Young RC: Threshold methotrexate concentration for in vivo inhibition of DNA synthesis in normal and tumorous target tissues. J Clin Invest 52: 1804-1811, 1973

8) Erttmann R, Landbeck G: Effect of oral cholestyramine on the elimination of high-dose methotrexate. $J$ Cancer Res Clin Oncol 110: 48-50, 1985

9) Gadgil SD, Damle SR, Advani SH, Vaidya AB: Effect of activated charcoal on the pharmacokinetics of high-dose methotrexate. Cancer Treat Rep 66: 1169-1171, 1982

10) Honda $Y$, Nakano M: Studies on adsorption characteristics of bile acids and methotrexate to a new type of anion-exchange resin, colestimide. Chem Pharm Bull (Tokyo) 48: 978-981, 2000

11) Huffman DH, Wan SH, Azarnoff DL, Hogstraten B: Pharmacokinetics of methotrexate. Clin Pharmacol Ther 14: 572-579, 1973

12) Leme PR, Creaven PJ, Allen LM, Berman M: Kinetic model for the disposition and metabolism of moderate and high-dose methotrexate (NSC-740) in man. Cancer Chemother Rep 59: 811-817, 1975

13) Lui CY, Lee MG, Chiou WL: Clearance studies of methotrexate in dogs after multiple-rate infusion. Cancer Res 45: 1545-1548, 1985

14) Shinozaki T, Watanabe H, Tomidokoro R, Yamamoto K, Horiuchi R, Takagishi K: Successful rescue by oral cholestyramine of a patient with methotrexate nephrotoxicity: nonrenal excretion of serum methotrexate. Med Pediatr Oncol 34: 226-228, 2000

15) Stoller RG, Jacobs SA, Drake JC, Lutz RJ, Chabner BA: Pharmacokinetics of high-dose methotrexate (NSC-740). Cancer Chemother Rep 6: 19-24, 1975

Address reprint requests to: K. Makino, M.D., Department of Neurosurgery, Kumamoto University School of Medicine, 1-1-1 Honjo, Kumamoto 860-8556, Japan. e-mail: kmakino@fc.kuh.kumamoto-u.ac.jp 